\section{AB012. Surgical management of malignant pleural mesothelioma}

\section{David Waller}

Department of Thoracic Surgery, St Bartholomew's Hospital, London, UK

Correspondence to: David Waller, FRCS(CTh). Consultant Thoracic Surgeon, St Bartholomew's Hospital, London, UK.

Email: david.waller1@nhs.net.

\begin{abstract}
The clinical suspicion of mesothelioma should be aroused when a patient presenting with a unilateral pleural effusion and pleural thickening gives a history of asbestos exposure. This maybe as a direct exposure whilst working with the substance or indirectly via environmental exposure. In most a thoracic CT is useful leading to an image guided pleural biopsy (if predominantly pleural thickening) or pleural aspiration (if predominantly effusion) to exclude adenocarcinoma. Effusion control can be achieved together with a definitive histological diagnosis by video assisted thoracoscopy (VATS), which does not necessarily need general anaesthesia. If the lung expands on effusion drainage then talc pleurodesis should be performed after biopsy; if the lung is entrapped then an indwelling pleural catheter should be inserted. If the patient is to proceed to further radical treatment then in general they should have similar cardiorespiratory reserve to withstand a lobectomy for lung cancer. Anaemia, leucocytosis and thrombocythaemia are poor prognostic indicators as is severe weight loss. It may be difficult to measure dynamic and static lung volumes due to the disease itself but differential lung perfusion imaging and echocardiographic assessment of ventricular function are useful, particularly if pneumonectomy is proposed. Since radical surgery should always be considered as part of multimodality therapy then the patient should have adequate renal function. The diagnostic biopsy must be sufficient for the tumour cell type to be identified. In most specialist centres pure sarcomatoid malignant pleural mesothelioma (MPM) precludes radical surgery. For biphasic disease the position is less clear and the proportion of sarcomatoid: epithelioid may be considered. The objective of radical surgery is to
\end{abstract}

achieve macroscopic complete resection (MCR) which means leaving no visible residual tumour or a $\mathrm{R} 2$ resection. Therefore, CT is used to exclude T4 disease (in conjunction with MRI) and CTPET may be used to investigate potential peritoneal M1 disease. Tumour thickness and volume on CT and avidity on CTPET may all be used to predict prognosis. As not all nodal stations can be sampled preoperatively then $\mathrm{N} 1$ disease is a relative contraindication to resection. Endoscopic ultrasound or mediastinoscopy can be used to exclude contralateral N3 disease. Where indicated by suspicious imaging, contralateral video assisted thoracoscopy or laparoscopy may be needed to exclude M1 disease. Currently we offer radical surgery in patients with N1 disease (providing MCR can be achieved) within the MARS2 trial but we await the results in order to inform future practice. In those with an entrapped lung after drainage a debulking VATS visceral decortication (partial pleurectomy) may be considered. This may improve survival but is intended to improve quality of life by improving dyspnoea and removing the need for drainage. Whilst MCR of the pleura is the objective this may require resection of the lung parenchyma due to visceral pleural invasion. Wedge resection is often performed; lobectomy rarely and pneumonectomy is almost never required. Pleurectomy/ decortication (PD) is the ideal outcome but in many cases an extended PD (EPD) is required due to pleural invasion of the muscle of the diaphragm. Every effort should be made to separate the diaphragmatic pleura from muscle but clearance should not be compromised nor should a potentially damaged and dysfunctional muscle be preserved. Total visceral pleurectomy is another potential area where clearance may be compromised as early disease is difficult to dissect from the underlying parenchyma. We advocate total pleurectomy since microscopic invasion may be present in macroscopically normal pleura and intraoperative frozen section analysis is unreliable. A systematic lymph node dissection is always performed to insure accurate staging. Together with the stations dissected in lung cancer, nodes in the internal mammary chain, pericardial, diaphragmatic and posterior intercostal nodes should be sampled. How radical surgery best fits into multimodality therapy remains to be determined. Radical hemithorax irradiation is promoted in the SMART protocol and attempts are being made to develop lung sparing protocols. The timing of systemic chemotherapy is still undetermined. Neoadjuvant chemotherapy does ensure that the patient receives all of the prescribed cycles since the recovery from radical surgery can compromise patient fitness and the ability to 
tolerate chemotherapy. However, as the response rates are under $50 \%$, there is a risk that tumour progression may lead to unresectability. The use of intrapleural cytotoxic chemotherapy after radical resection has many proponents but is not universally used and lacks high grade supportive evidence. The MARS2 trial in the UK of EPD + chemotherapy $v s$. chemotherapy alone should complete the recruitment target of 326 patients by early 2020 and therefore report in 2022. Until then we should concentrate on standardizing the surgical technique and how we report intraoperative results and completeness of resection.

Keywords: Malignant pleural mesothelioma (MPM); surgery; pleurectomy/decortication (PD)

doi: $10.21037 /$ shc.2019.AB012

Cite this abstract as: Waller D. Surgical management of malignant pleural mesothelioma. Shanghai Chest 2019;3:AB012. 